\title{
BER Analysis of OSTBC in MIMO using ZF \& MMSE Equalizer
}

\author{
Abhijit Singh Thakur \\ Scholar, ECE, IPS Academy, Indore, India \\ Prof. Nitin jain \\ Prof, ECE, IPS Academy, Indore, India
}

\begin{abstract}
In this paper, a bit error rate analysis is analyzed for multiple-input-multiple-output (MIMO) system with finite-bit feedback is considered in PSK modulation technique, where a transmit signal consists of a rotational precoder followed by an orthogonal space-time block code (OSTBC) which achieve full diversity when a linear receiver, such as, zero forcing (ZF) or minimum mean square (MMSE), is used. By choosing different parameters, codes with different symbol rates and orthogonally can be obtained.In this paper, we compare the performance of a family of space-time codes. Simulations show how the pre-coders obtained by our proposed criterion and method perform better bit error rate reduction compared to the existing ones.
\end{abstract}

Index Term- Multiple-input-Multiple output (MIMO) systems, MMSE and ZF equalizer, Orthogonal space time block code(OSTBC)

\section{INTRODUCTION}

A Multiple-Input Multiple-Output (MIMO) system is one of the most significant technical breakthroughs in modem communication system. MIMO systems are simply defined as the containing multiple transmitter antennas and multiple receiver antennas. Communication part show that MIMO systems can provide a potentially very high capacity, in many cases, grows approximately linear with the number of antennas. MIMO systems have already been implemented in wireless communication systems. Capacity limits of the Gaussian multiuser broadcast channel with multiple transmit antennas at the base station and multiple receive antennas at each user have captured a large amount of research in recent years [1]. Rotational pre-coding was originally proposed as a preequalization technique for channels with inter-symbol-interference. The operation relies critically on the availability of channel state information (CSI) in order to accurately subtract the interference that otherwise would be created at each decentralized receiver. Broadcast channels have been proposed for OSTBC pre-coder, including zero-forcing designs and minimum mean square error (MMSE) designs [2]. Multiple, combination of OSTBC precoded signals could be an efficient solution to support diversity fairness without affecting the best-ordered users [3]. In case former can be used without Channel State Information (CSI) at the transmitter and allows mitigation of fading and exploitation of transmit-receive diversity. CSI is known at the transmitter, higher throughput can be attained using spatial multiplexing, can be implemented as multi beam transmit beam forming[4].Almouti precoding is a transmitter equalization technique where equalization is performed at the transmitter side, It can eliminate error propagation by moving the FBF of DFE to the transmitter and allow us to use current capacityachieving channel codes[5].Algorithm are designed for a minimum mean square error (MMSE) approach under a constraint on the overall transmit power. The solution to this problem requires a large number of matrix inversions (equal to the number of active users) and may be unfeasible when applied to heavy-loaded systems. All matrix inversions are replaced by a single factorization [6]. In a noise free case, zero forcing corresponds to bringing down the ISI to zero. ISI is significant compared to noise this will be useful. Frequency response F(f) the zero forcing equalizer $\mathrm{C}(\mathrm{f})$ is constructed such that $\mathrm{C}(\mathrm{f})=1 / \mathrm{F}(\mathrm{f})$. Combination of channel and equalizer gives a flat frequency response and linear phase $\mathrm{F}(\mathrm{f}) \mathrm{C}(\mathrm{f})=1$. Than response of a particular channel is $\mathrm{H}(\mathrm{s})$ then the input signal is multiplied by the reciprocal of this[7]. The concept behind OSTBC pre-coding is a transmitter equalization technique, elimination of the ISI of our channel and focus on ZF and MMSE to Achieve better transmit diversity. 


\section{ORTHOGONAL SPACE-TIME BOCK CODING}

To minimize decoding complexity, space time block code (STBC) has been discovered. The orthogonal design of this scheme linear processing at the receiver. In this paper, emphasis within space-time coding is placed on block approaches. STBC based on orthogonal design obtains full diversity gain with low decoding complexity therefore is widely used. An OSTBC1 matrix is composed of linear combinations of constellation symbols $s_{1}$, $s_{2}, \ldots, s_{y}$ and their conjugates, and encoding therefore only requires linear processing. The most important special case is the Alamouti codes for two transmit antennas. It is used to achieve space-time transmit diversity (STTD), and has been adopted in several third generation $(3 \mathrm{G})$ cellular standards because it maximizes diversity gain. The code matrix for OSTBC satisfies for all complex code symbols. For example, the Alamouti code is an OSTBC with 2 transmit antennas, for which the transmit matrix i.e., a pair of emblems $\mathrm{s}^{*} 1$ in addition to $\mathrm{s}^{*} 2$ in addition to their own conjugates usually are carried over a pair of moment slot machine games. With initially slot machine game, s1 in addition to s2 usually are carried on the antenna 1 in addition to 2, respectively; throughout the following mark time period, $-\mathrm{s} * 2$ is carried on the antenna 1 , in addition to $\mathrm{s}^{*} 1$ is on the antenna 2. Much more normal OSTBC set ups usually are discussed throughout. As an illustration, signal matrices with regard to rate $1 / 2$ in addition to $3 / 4$ signal making use of a number of antenna receive by simply.

$$
\mathrm{S}=\left[\begin{array}{cc}
s_{1} & s_{2} \\
-s_{2}^{*} & s_{1}^{*}
\end{array}\right]
$$

At each time slot, a column of the codeword matrix is transmitted across different antennas. At the end of a block, the receiver employs zero forcing (ZF) and minimum mean square Error (MMSE) decoding to separate different transmitted symbols contained in a codeword.

\section{LIMITED FEEDBACK PRE-CODING}

You're pre-coding approaches described preceding was being according to possessing perfect funnel express info at the transmitter. On the other hand, in real systems, receivers may just responses quantized info which is described by a confined volume of bits. If your identical pre-coding approaches are employed, nevertheless currently according to wrong funnel info, additional disturbance looks. That is an example in confined responses pre-coding. The received signal in multi-user MIMO with limited feedback pre-coding is mathematically described as

$y_{k}=h_{k}^{M} \sum_{i=1}^{\bar{k}} w_{i s_{i}}+n_{k}$

In this case, the beam-forming vectors are distorted as

$$
w_{i}^{n}=s_{i}+w_{i}
$$

Where $w_{i}$ is the optimal vector and $e_{i}$ is the error vector caused by inaccurate CSI (channel state information). The received signal can be written as

$y_{h=h} h_{i=1}^{k} \sum_{i}^{k} s_{i}+h_{n}^{k} \sum_{i=1}^{k} e_{i} s_{i}+n_{k}$

Where $\mathrm{k}=1,2,3 \ldots$

$h_{k}^{k} \sum_{i=1}^{k} w_{i} s_{i}$ 
Where is the additional interference at user $\mathrm{K}$ according to the limited feedback pre-coding[7]. To reduce this interference need higher accuracy in the channel state information feedback is required, which is to turn reduces the throughput in the process of uplink.

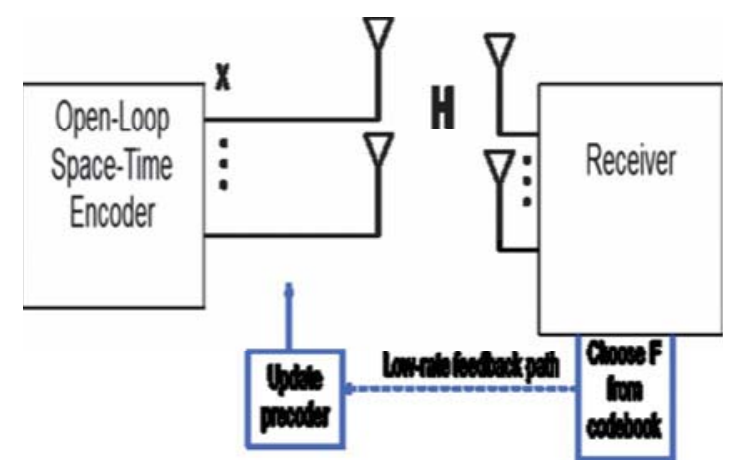

Fig1. Limited Feedback Pre-coding.

\section{EQUALIZER}

Equalizer provides an approximate inverse of channel frequency response because is a digital filter. Equalization decrease to effect of ISI probability of error that occurs without suppression of ISI, noise power enhancement, reduction of ISI effects has to be balanced. In our paper zero forcing and MMSE equalizer is used[8].

\subsection{MMSE \& Zero Forcing Equalizer.}

The term equalization is used to describe any signal processing operation that minimize or a compensate inter symbol interference (ISI) created by multipath with time dispersive channels $\left(\mathrm{W}>\mathrm{B}_{\mathrm{C}}\right)$.

Basically equalization is a technique is used to improve receiver signal quality.Equalizer must be "adaptive", since channels are time varying. There are 2 types of equalization.

1. Linear equalization

2. Non linear equalization

A couple of operation modalities on an adaptive equalizer: coaching and also tracking. Several aspects have an effect on enough time comprising in excess of that a equalizer converges: equalizer algorithm, equalizer construction and also time period charge associated with adjust with the multipath airwaves funnel. TDMA instant methods usually are especially well suited for equalizers. Equalizer is usually implemented at baseband or at IF in a receiver

$$
\mathrm{Y}(\mathrm{t})=\mathrm{x}(\mathrm{t}) * f^{*}(\mathrm{t})+n_{b}(\mathrm{t})
$$

$f^{*}(t)$ :it is the complex conjugate of $f(t)$

$\mathrm{n}_{\mathrm{b}}(\mathrm{t})$ :it is the baseband noise at the input of the equalizer

$h_{e q}(t): i t$ is the impulse response of the equalizer

Block diagram- this block diagram show the communication system with adaptive equalizer at receiver 


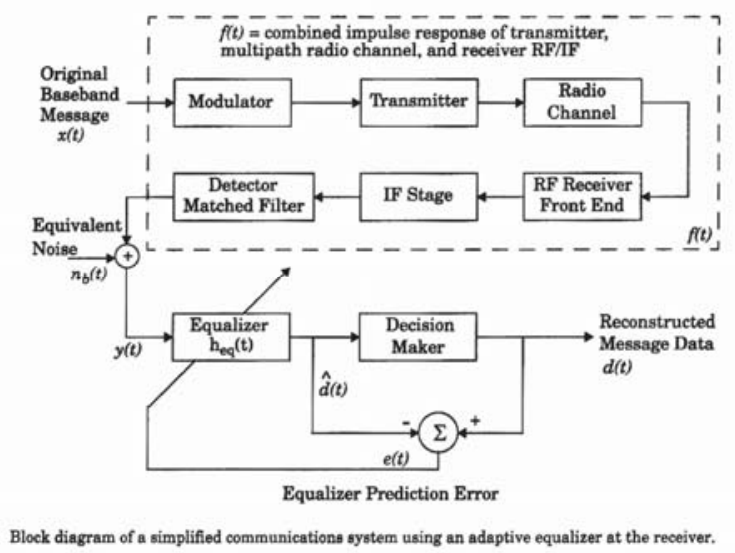

Fig 2

In Fig. 1, if $d(t)$ is not the feedback path to adapt the equalizer, the equalization is linear In Fig. 1, if $d(t)$ is feed back to change the subsequent outputs of the equalizer, the equalization is nonlinear in nature .

If the channel is frequency selective, the equalizer increases the frequency components with small amplitudes and attenuates the strong frequencies in the received frequency response. For a time-varying channel, an adaptive equalizer is needed to track the channel variations.

\section{ZERO FORCING EQULIZER}

Zero Forcing Equalizer in communication systems, work on inverts the frequency response of the channel. ZF applies the inverse of the channel to the received signal, to restore the signal before the channel. The name is Zero Forcing called because, bringing down the ISI to zero in a noise free case. ISI is significant compared to noise, ZF is very useful. Frequency response $F(f)$ the zero forcing equalizer $C(f)$ is constructed such that $C(f)=1 / F(f)$. Channel is combined than flat frequency response and linear phase $F(f) C(f)=1 . H(s)$ is represented channel response for a particular channel is multiplied by the reciprocal of this. Remove the effect of channel from the received signal, in particular the Inter symbol Interference (ISI)[7]. Let us consider 2x2 MIMO channel, than channel is modeled as

The received signal on the first receive antenna

$y_{1}=h_{1,1} x_{1}+h_{1,2} x_{2}+n_{1}=\left[h_{1,1} h_{1,2}\right]+\left[\begin{array}{l}x_{1} \\ x_{2}\end{array}\right]+n_{1}$

The received signal on the Second receive antenna

$y_{2}=h_{2,1} x_{1}+h_{2,2} x_{2}+n_{2}=\left[h_{2,1} h_{2,2}\right]+\left[\begin{array}{l}x_{1} \\ x_{2}\end{array}\right]+n_{2}$

Where

$y_{1}, y_{2}$ are the received signal on the first and second antenna respectively,

$h_{1,1}$ Is the channel from 1 st transmit antenna to 1 st receive antenna,

$h_{1,2}$ Is the channel from 2 nd transmit antenna to 1 st receive antenna,

$h_{2,1}$ Is the channel from 1 st transmit antenna to 2 nd receive antenna,

$h_{2,2}$ Is the channel from 2 nd transmit antenna to 2 nd receive antenna, 
$\mathrm{x}_{1}, \mathrm{x}_{2}$ are the transmitted symbols and

$\mathrm{n}_{1}, \mathrm{n}_{2}$ are the noise on 1 st and 2 nd receive antennas.

The equation can be represented in matrix notation as follows

$\left(\begin{array}{l}y_{1} \\ y_{2}\end{array}\right)=\left(\begin{array}{ll}h_{1,1} & h_{1,2} \\ h_{2,1} & h_{2,2}\end{array}\right)\left(\begin{array}{l}x_{1} \\ x_{2}\end{array}\right)+\left(\begin{array}{l}h_{1} \\ n_{2}\end{array}\right)$

Equation is

$$
\mathrm{Y}=\mathrm{H} \cdot \mathrm{x}+\mathrm{n}
$$

To solve for $\mathrm{x}$, we need to find a matriix W which satisfies WA=I the Zero Forcing (ZF) detector for

$\mathrm{W}=(\mathrm{AAA})-1 \mathrm{AA}$

Where A - Channel Matrix

W - Equalization Matrix

Note that the off diagonal elements in the matrix AAA are not zero, because the off diagonal elements are non zero in values. Zero forcing equalize performing well but is not the best equlizer[7]. It is simple way and easy to implement.BPSK Modulation use Rayleigh fading channel, the BER is defined as

$$
\mathrm{Pb}=\frac{1}{2}\left(1-\sqrt{\frac{(\vec{b})}{(E b / N o)+1}}\right)
$$

Where

$\mathrm{Pb}$ - Bit ErrorRate

$\mathrm{Eb} /$ No -Signal to noise Ratio

\section{MMSE (MINIMUM MEAN SQUARE ERROR) EQULIZER}

A minimum mean square error (MMSE) estimator perfume, describes the approach which minimizes the mean square error (MSE), Common measure of estimator quality. Output of system, MMSE equalizer is that it does not usually eliminate ISI completely but, minimizes the total power of the noise and ISI components in output. Let $\mathrm{x}$ is define an unknown random variable, and let $y$ is define a known random variable[7]. Measurement of y than estimator $\mathrm{x}^{\wedge}(\mathrm{y})$ is any function, mean square error is

$$
\mathrm{MSE}=\mathrm{E}\left\{\left(\mathrm{X}^{\wedge}(\mathrm{y})-\mathrm{X} 2\right)\right\}
$$

Expectation is taken over both $\mathrm{x}$ and $\mathrm{y}$. An estimator achieving minimal MSE[7]. In many different cases, it is not possible to determine a much closed form for the MMSE estimator. So in these cases, one possibility is to seek the technique minimizing the MSE within a particular class, and this is a class of linear estimators. MMSE estimator is the estimator achieving minimum MSE among all estimators of the form AY $+b$. Measurement $\mathrm{Y}$ is a random vector, $\mathrm{A}$ is defining a matrix and $\mathrm{b}$ is defining a vector. Let us now try to understand the math for extracting the two symbols which interfered with each other[7]. In the first time slot, the received signal on the first receive antenna is,

$y_{1}=h_{1,1} x_{1}+h_{1,2} x_{2}+n_{1}=\left[h_{1,1} h_{1,2}\right]+\left[\begin{array}{l}x_{1} \\ x_{2}\end{array}\right]+n_{1}$

The received signal on the Second receive antenna is, 
$y_{2}=h_{2,1} x_{1}+h_{2,2} x_{2}+n_{2}=\left[h_{2,1} h_{2,2}\right]+\left[\begin{array}{l}x_{1} \\ x_{2}\end{array}\right]+n_{2}$

Where

$y_{1}, y_{2}$ are the received signal on the first and second antenna respectively,

$h_{1,1}$ Is the channel from 1 st transmit antenna to 1 st receive antenna,

$h_{12}$ Is the channel from 2 nd transmit antenna to 1 st receive antenna,

$h_{2,1}$ Is the channel from 1 st transmit antenna to 2 nd receive antenna,

$h_{2,2}$ Is the channel from 2 nd transmit antenna to 2 nd receive antenna,

$\mathrm{x}_{1}, \mathrm{x}_{2}$ are the transmitted symbols and

$\mathrm{n}_{1}, \mathrm{n}_{2}$ are the noise on 1 st and $2 \mathrm{nd}$ receive antennas.

The equation can be represented in matrix notation as follows

$$
\left(\begin{array}{l}
y_{1} \\
y_{2}
\end{array}\right)=\left(\begin{array}{ll}
h_{1,1} & h_{1,2} \\
h_{2,1} & h_{2,2}
\end{array}\right)\left(\begin{array}{l}
x_{1} \\
x_{2}
\end{array}\right)+\left(\begin{array}{l}
n_{1} \\
n_{2}
\end{array}\right)
$$

Equivalently,

$$
\mathrm{y}=\mathrm{H} . x+\mathrm{n}
$$

The Minimum Mean Square Error (MMSE) approach tries to[7] find a coefficient W which minimizes the

$$
\mathrm{E}\{[\mathrm{Wy}-\mathrm{x}\}[\mathrm{Wy}-\mathrm{x}] \mathrm{H}\}
$$

To solve for $\mathrm{x}$, we need to find a matrix $\mathrm{W}$ which satisfies $\mathrm{WH}=\mathrm{I}$

Where

y- Received signal

H - Channel Matrix and

W - Equalization Matrix

$\mathrm{n}$ - Channel noise

\section{SIMULATION RESULTS}

STBC pre-coding is performed for various number of transmit and receive antennas in order to achieve the better performance mainly focuses on receiver is equipped with single antenna where exists only the transmit diversity, but without any receive diversity. Zero forcing (ZF) and minimum mean square error (MMSE) algorithm is used, Zero forcing (ZF) work which invert the frequency response of the channel and (MMSE) equalizer is that it does not usually eliminate ISI completely but, minimizes the total power of the noise and ISI components in the output. is useful to achieve better transmit diversity. 


\subsection{COMPARISON OF OSTBC AND PRECODED OSTBC WITH MMSE EQUALIZER}

The system is designed for four transmit antenna and four receive antennas for a packet size of 10000 bits. The QPSK modulation is being used here in Rayleigh fading environment. Signal-to-noise ratio (SNR) is ranging from 0 to $25 \mathrm{db}$.

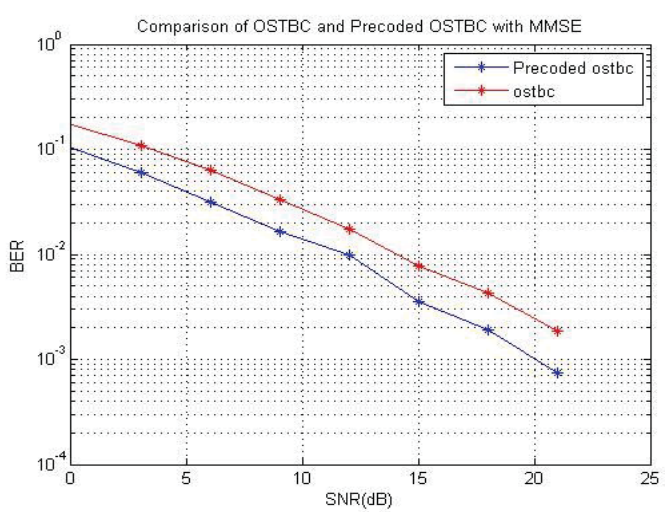

Fig No.7.1 BER Performance of OSTBC and pre-coded OSTBC with MMSE Equalizer

The above graph shows the performance of OSTBC and pre-coded OSTBC using MIMO $4 \times 4$ (four transmit antenna and four receive antennas) with MMSE equaliser system. Here the value of bit error rate (BER) is decreases exponentially when the value of SNR increases. The maximum value of BER is $10^{-0.8}$ for $\mathrm{SNR}=0 \mathrm{db}$ for MIMO $4 \times 4$, which decreases to $10^{-2.9}$ for OTBC and MIMO $4 \times 4$ decreases to $10^{-3.4}$ for Pre-coded OSTBC with MMSE at $\mathrm{SNR}=25 \mathrm{db}$.

\begin{tabular}{|c|c|c|}
\hline $\begin{array}{c}\text { SIGNAL- } \\
\text { TO- }\end{array}$ & MINIMUM -MEAN & MINIMUM- \\
NOISE & ERROR(MMSE)WITH & MEAN \\
RATIO & PRECODED OSTBC & ERROR \\
(SNR) & (BER) & (MMSE) \\
& & WITHOUT \\
& & OSTBC \\
& & PRECODER \\
& & (BER) \\
\hline $0 \mathrm{db}$ & 0.1066 & 0.1675 \\
\hline $3 \mathrm{db}$ & 0.0578 & 0.1128 \\
\hline $6 \mathrm{db}$ & 0.0323 & 0.0667 \\
\hline $9 \mathrm{db}$ & 0.0166 & 0.0667 \\
\hline $12 \mathrm{db}$ & 0.0072 & 0.0356 \\
\hline $15 \mathrm{db}$ & 0.0041 & 0.0187 \\
\hline $18 \mathrm{db}$ & 0.0025 & 0.0045 \\
\hline $21 \mathrm{db}$ & 0.0145 & \\
\hline & & \\
\hline
\end{tabular}




\subsection{COMPARISON OF OSTBC AND PRECODED OSTBC WITH ZF EQUALIZER}

The system is designed for four transmit antenna and four receive antennas for a packet size of 10000 bits. We use code length of 64 for QPSK modulation used here in Rayleigh fading environment. Signal-to-noise ratio (SNR) is ranging from 0 to $25 \mathrm{db}$.

The below graph shows the performance of MIMO for $4 \times 4$ with four transmit antenna and four receive antennas. Here the value of bit error rate (BER) is decreases exponentially when the value of SNR increases.

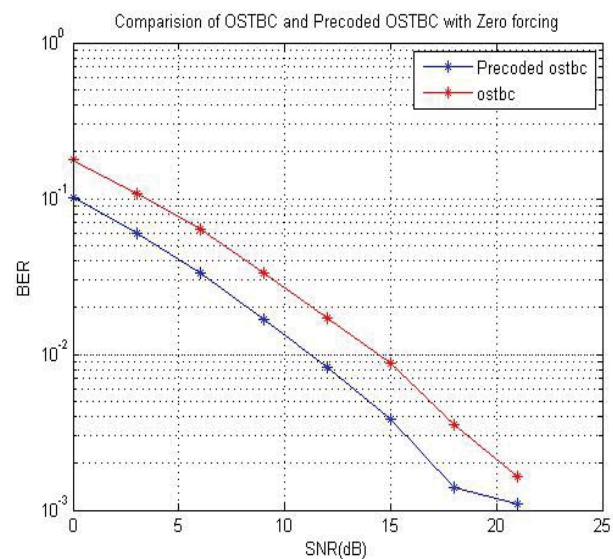

Fig 7.2 Comparison of BER Performance of OSTBC and Pre-coded OSTBC With ZF Equalizer

The maximum value of BER is $10^{-0.8}$ for $\mathrm{SNR}=0 \mathrm{db}$ for OSTBC MIMO $4 \times 4$ with $\mathrm{ZF}$ equalizer and maximum value of is $10^{-2.8} \mathrm{SNR}=0$ which decreases to $10^{-3.0}$ for pre-coded OSTBC MIMO $4 \times 4$ with $\mathrm{ZF}$ at $\mathrm{SNR}=25 \mathrm{db}$.

\begin{tabular}{|c|c|c|}
\hline $\begin{array}{c}\text { SIGNAL-TO-NOISE RATIO } \\
\text { (SNR) }\end{array}$ & $\begin{array}{c}\text { PRECODED OSTBC WITH ZF } \\
\text { (BER) }\end{array}$ & $\begin{array}{c}\text { PRECODED OSTBC } \\
\text { WITHOUT ZF (BER) }\end{array}$ \\
\hline $0 \mathrm{db}$ & 0.1067 & 0.1674 \\
\hline $3 \mathrm{db}$ & 0.0578 & 0.1129 \\
\hline $6 \mathrm{db}$ & 0.0324 & 0.0667 \\
\hline $9 \mathrm{db}$ & 0.1665 & 0.0356 \\
\hline $12 \mathrm{db}$ & 0.0073 & 0.0187 \\
\hline $15 \mathrm{db}$ & 0.0041 & 0.0076 \\
\hline $18 \mathrm{db}$ & 0.0025 & 0.0027 \\
\hline $21 \mathrm{db}$ & 0.0014 & 0.0027 \\
\hline
\end{tabular}

Table No.7.2 Comparison of BER Performance of OSTBC and Pre-coded OSTBC With ZF Equalizer 


\subsection{COMPARISON OF OSTBC AND ALAMAUTI PRECODING WITH MMSE EQUALIZER}

The system is designed for two transmit antenna and two receive antennas for a packet size of 10000 bits. We use code length of 16 for BPSK modulation used here in Rayleigh fading environment. Signal-to-noise ratio (SNR) is ranging from 0 to $25 \mathrm{db}$.

The below graph shows the performance of MIMO. Here the value of bit error rate (BER) is decreases exponentially when the value of SNR increases.

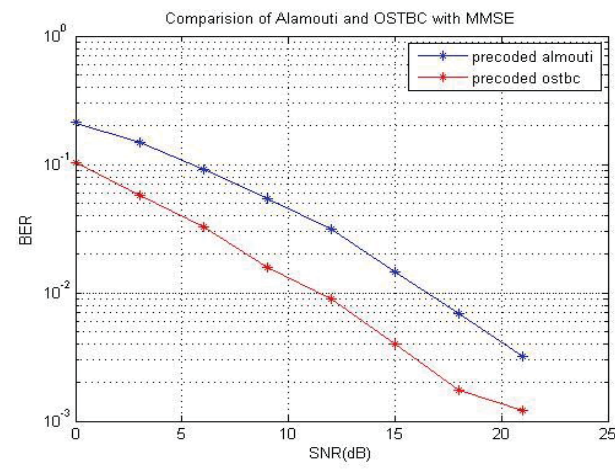

Fig No. 7.3 Comparison of BER Performance OSTBC and Alamauti Pre-coding With MMSE Equalizer

The maximum value of $\mathrm{BER}$ is $10^{-0.9}$ for $\mathrm{SNR}=0 \mathrm{db}$ for pre-coded Alamauti code MIMO $2 \times 2$ with MMSE Equalizer which decreases to $10^{-2.8}$ and pre-coded OSTBC MIMO $2 \times 2$ decrease it to $10^{-3.0}$ with MMSE equalizer at $\mathrm{SNR}=25 \mathrm{db}$.

\begin{tabular}{|c|c|c|}
\hline $\begin{array}{c}\text { SIGNAL-TO-NOISE RATIO } \\
\text { (SNR) }\end{array}$ & $\begin{array}{c}\text { PRECODED ALMOUTI } \\
\text { WITH MINIMUM MEAN } \\
\text { SQURE ERROR (BER) }\end{array}$ & $\begin{array}{c}\text { PRECODED OSTBC WITH } \\
\text { MNIMUM SQURE } \\
\text { ERROR(BER) }\end{array}$ \\
\hline $0 \mathrm{db}$ & 0.2150 & 0.1001 \\
\hline $3 \mathrm{db}$ & 0.1415 & 0.0584 \\
\hline $6 \mathrm{db}$ & 0.0891 & 0.0299 \\
\hline $9 \mathrm{db}$ & 0.0526 & 0.0167 \\
\hline $12 \mathrm{db}$ & 0.0272 & 0.0093 \\
\hline $18 \mathrm{db}$ & 0.0145 & 0.0041 \\
\hline $12 \mathrm{db}$ & 0.0074 & 0.0021 \\
\hline $14 \mathrm{db}$ & 0.0041 & 0.0009 \\
\hline
\end{tabular}

Table No.7.3 Comparison of BER Performance OSTBC and Alamauti Pre-coding With MMSE Equalizer

\subsection{COMPARISON OF OSTBC AND ALAMAUTI PRECODING WITH ZF EQUALIZER}

The system is designed for two transmit antenna and two receive antennas for a packet size of 10000 bits. We use code length of 16 for BPSK modulation used here in Rayleigh fading environment. Signal-to-noise ratio (SNR) is ranging from 0 to $25 \mathrm{db}$. 
The below graph shows the performance of MIMO. Here the value of bit error rate (BER) is decreases exponentially when the value of SNR increases.

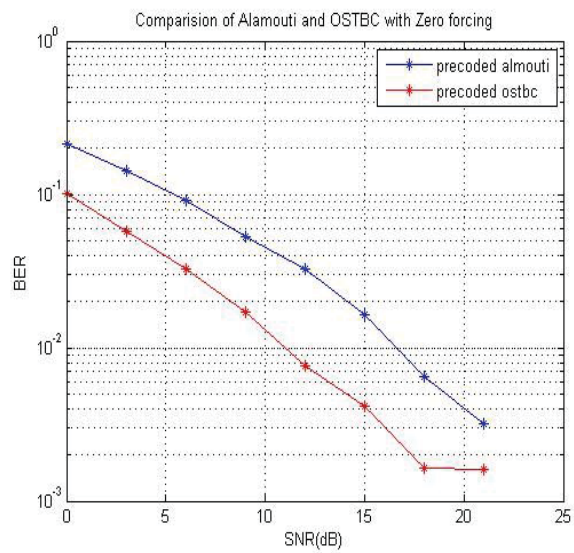

Fig No. 7.4 Comparison of BER Performance of OSTBC and Alamouti Pre-coding with ZF Equalizer

The maximum value of BER is $10^{-0.9}$ for pre-coded Alamoauti code at $\mathrm{SNR}=0 \mathrm{db}$ of MIMO $2 \times 2$ with $\mathrm{ZF}$ equalizer and maximum value is $10^{-2.7}$ which decreases to $10^{-3.0}$ for pre-coded OSTBC MIMO $2 \times 2$ with $\mathrm{ZF}$ equalizer at $\mathrm{SNR}=25 \mathrm{db}$.

\begin{tabular}{|c|c|c|}
\hline $\begin{array}{c}\text { SIGNAL-TO-NOISE RATIO } \\
(\text { SNR) }\end{array}$ & $\begin{array}{c}\text { ZERO-FORCING (ZF) WITH } \\
\text { PRECODED ALMAOUTI } \\
\text { (BER) }\end{array}$ & $\begin{array}{c}\text { ZERO- FORCING(ZF) } \\
\text { WITH PRECODED } \\
\text { OSTBC(BER) }\end{array}$ \\
\hline $0 \mathrm{db}$ & 0.2150 & 0.1020 \\
\hline $3 \mathrm{db}$ & 0.1415 & 0.0600 \\
\hline $6 \mathrm{db}$ & 0.0891 & 0.0329 \\
\hline $9 \mathrm{db}$ & 0.0526 & 0.0175 \\
\hline $12 \mathrm{db}$ & 0.0272 & 0.0088 \\
\hline $15 \mathrm{db}$ & 0.0145 & 0.0039 \\
\hline $18 \mathrm{db}$ & 0.0074 & 0.0024 \\
\hline $21 \mathrm{db}$ & 0.0041 & 0.0014 \\
\hline
\end{tabular}

Table No.7.4 Comparison of BER Performance OSTBC and Alamauti Pre-coding With ZF Equalizer

\section{REFERENCES}

[1] G. Caire and S. Shamai, "On the achievable throughput of a multiantenna Gaussian broadcast channel," IEEE Trans. Inf. Theory, vol. 4, pp. 1691-1706, Jul. 2003.

[2] C. Windpassinger, R. F. H. Fischer, T. Vencel, and J. B. Huber, "Precoding in multiantenna and multiuser communications," IEEE Trans. Wireless Commun., vol. 3, pp. 1305-1316, Jul. 2004.

[3] A. Tomasoni, M. Ferrari, S. Bellini, and G. Caire, "Effective beamforming and efficient scheduling for multi-user MIMO broadcast channels," in Proc. IEEE Inf. Theory Workshop, Taormina, Italy, Oct. 2009, pp. 520-523.

[4] G. Dimic and N. D. Sidiropoulos, "On downlink beamforming with greedy user selection: Performance analysis and a simple new algorithm,” IEEE Trans. Signal Process., vol. 53, pp. 3857-3868, Oct. 2005.

[5] Tomlinson Harashima Precoding for Multi-Gigabit short-haul Transmission over Plastical Optical Fibers, Laura Caballero, Nadales September 7, 2010.

[6] Non-Linear Pre-Coding for Multiple-Antenna Multi-User Downlink Transmissions with Different QoS Requirements Luca Sanguinetti, Member, IEEE, and Michele Morelli, Member, IEEE. 
[7] Performance analysis of BPSK system with ZF \& MMSE equalization, Manish Kumar Department of Electronics and Communication Engineering Swift institute of Engineering \& Technology, Rajpura, Punjab, India.

[8] Minimum SER Zero-Forcing Transmitter Design for MIMO Channels with Interference Pre-subtraction Michael Botros Shenouda, Student Member, IEEE, and Timothy N. Davidson, Member, IEEE.

[9] R. Kwan, C. Leung, and J. Zhang, "Proportional fair multiuser scheduling in LTE,” IEEE Signal Process Lett., vol. 16, pp. 461-464, Jun. 2009.

[10] A. Molisch, Wireless Communications. Wiley-IEEE Press, 2005.

[11] D. Gesbert, M. Shafi, D. Shiu, P. Smith, and A. Naguib, “ an overview of MIMO space-time coded wireless systems," IEEE Journal on selected areas in Communications, vol. 21, no. 3, pp. 281-302, 2003.

[12] G. Tsoulos, MIMO system technology for wireless 